\title{
Extragalactic hard X-ray source counts with INTEGRAL observatory: A Progress Report
}

\author{
R. Krivonos ${ }^{1,2} \dagger$, M. Revnivtsev ${ }^{1,2}$ S. Sazonov ${ }^{1,2}$ E. Churazov ${ }^{1,2}$ \\ and R. Sunyaev ${ }^{1,2}$ \\ ${ }^{1}$ Space Research Institute, Moscow, Russia \\ ${ }^{2}$ Max-Planck Institut für Astrophysik, Garching, Germany
}

\begin{abstract}
We present results from the hard X-ray sky survey being performed with INTE$G R A L$ observatory. During two years of operation the significant sky area was covered with moderate sensitivity which now allows us to estimate surface number density of Active Galactic Nuclei (AGN) in hard (17-60 keV) energy band. Our catalog of detected sources comprises $>60$ AGN with wide range of intrinsic absorption. We used $24000 \mathrm{deg}^{2}$ sky coverage (high Galactic latitude observations) with total exposure $>2$ Msec to derive source number-flux relation. The estimated surface density of extragalactic hard X-ray sources with flux $>10^{-11} \mathrm{erg} \mathrm{s}^{-1} \mathrm{~cm}^{-2}$ is $(1.0 \pm 0.5) 10^{-2} \mathrm{deg}^{-2}$.
\end{abstract}

Keywords. Galaxies: active - Galaxies: Seyfert - Gamma rays: observations - X-rays: galaxies.

\section{Introduction}

Over the past few years there have been remarkable developments in our understanding of the evolution of active galactic nuclei. Extensive X-ray observations demonstrated that the space density of AGN with low X-ray luminosities peaks at lower redshift than that of AGN with high X-ray luminosities (Steffen et al., 2003; Ueda et al., 2003) which indicates that a substantial amount of the total black hole growth has occurred more recently than was suggested by optical surveys of powerful quasars (Boyle et al., 2000; Fan et al., 2001). The discovered tight correlation between the mass of the supermassive black hole and the properties of the galactic spheroid in which it resides (Magorrian et al., 2002; Tremaine et al., 2002) points to a close connection between the formation of SMBHs and that of their host galaxies.

Deep, small area X-ray surveys have been used for successive study of statistical properties of luminous active galactic nuclei at medium and high redshifts. However, the census of AGN in the more nearby Universe is far from being complete, in particular because most of the local AGN activity is believed to be highly obscured and also due to the difficulty of implementing the large area surveys.

We present a progress report on a hard X-ray survey of a large part of the sky performed with the INTEGRAL observatory.

\section{X-ray surveys}

Deep surveys performed in the standard X-ray band (2-10 keV) with grazing-incidence telescopes on ASCA (Ueda et al., 1999), BeppoSAX (Giommi et al., 2000), Chandra (Giacconi et al., 2002) and XMM-Newton (Hasinger et al., 2001) have provided a wealth of information about AGN at redshifts higher than $\backsim 0.3$. However, such surveys cover 


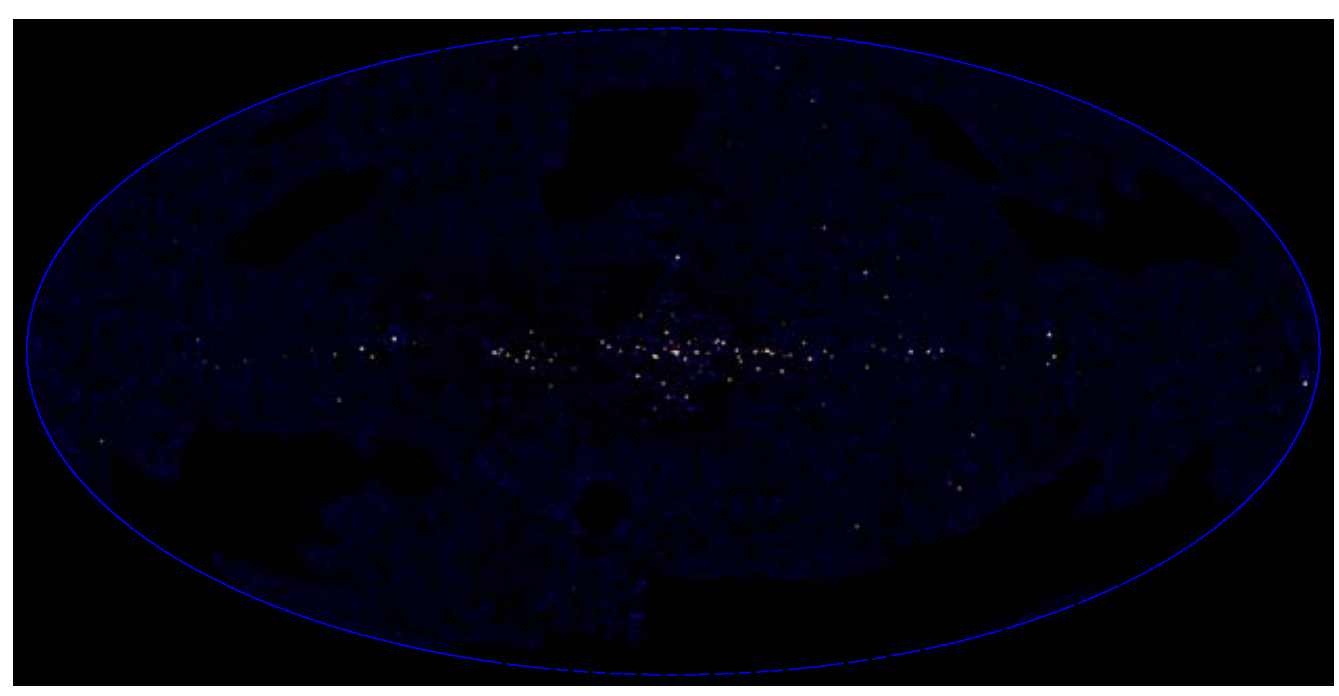

Figure 1. The current sky coverage by INTEGRAL observations. The significance map in $17-60 k e V$ energy band is shown.

small areas of the sky (at most 100 sq. deg) and cannot tell us much about the number density and luminosity distribution of nearby AGN $(z<0.1)$. Furthermore, there are hints that most of the local AGN are heavily obscured at optical and standard X-ray energies and therefore could only be observed in the far infrared or in hard X-rays.

In spite of development progress of AGN evolution at medium and high redshifts provided by small area X-ray surveys our current estimates of the number density and emissivity of local AGN are uncertain by a factor of a few, and removing this uncertainty is very important if one wishes to understand the evolution of the AGN population at low redshifts. There is therefore a clear need for very large sky area surveys performed above $10 \mathrm{keV}$, or even better $20 \mathrm{keV}$, i.e. at photon energies where the effect of photoabsorbtion of X-rays on neutral gas is dramatically reduced and thus practically unbiased statistics of AGN can be obtained.

\section{X-ray survey with INTEGRAL}

Before recently, our knowledge of the statistical properties of the local population of hard X-ray sources was based on observations performed for the whole sky in the 2-100 $\mathrm{keV}$ energy band more than 20 years ago by the the HEAO-1 observatory (Wood et al., 1984; Piccinotti et al., 1982; Levine et al., 1984). Recently, a new hard X-ray (3-20 keV) all-sky survey was performed by the RXTE observatory (Revnivtsev et al., 2004). This survey has detected a representative number of AGN, mostly nearby Seyfert galaxies, characterized by a range of absorbing column densities up to $N_{H}=10^{24} \mathrm{~cm}^{-2}$.

At present the all-sky survey in hard X-rays can be performed with wide-field telescopes on board INTEGRAL (Winkler et al., 2003) and Swift (Gehrels et al., 2004) observatories. The coded-aperture telescope BAT (Barthelmy et al., 2005) onboard Swift is sensitive in the 15-150 keV band and has very large (1.4sr, half-coded) field of view which make it possible to cover all sky in reasonable time. The Swift mission is operating from the end of 2004 and observational data is publicly available from April 2005. In this work we used INTEGRAL data obtained during two-years of observations. Among instruments onboard INTEGRAL, the IBIS telescope (Ubertini et al., 2003) with ISGRI (Lebrun et al., 2003) 

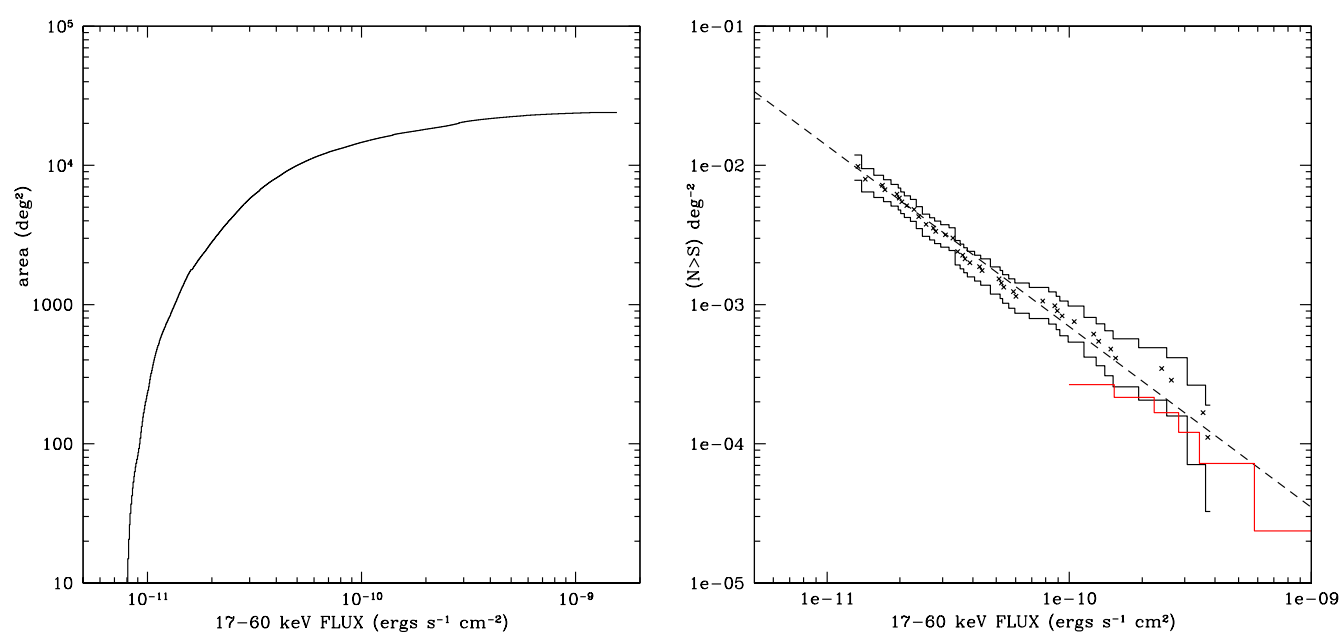

Figure 2. Left plot shows survey coverage area versus limiting flux. At the right, number-flux relation derived from extragalactic source sample (black data points) is demonstrated. For comparison HEAO-1 A4 source counts are also shown (red points).

detector is most suitable for hard X-ray survey, it has large $\backsim 30^{\circ} \times 30^{\circ} \mathrm{FOV}$, moderate angular resolution $\left(12^{\prime}\right)$ and $15-200 \mathrm{keV}$ operating energy range. Extragalactic fields observations demonstrated capability of IBIS/ISGRI to reach limiting flux of $<$ mCrab (Krivonos et al., 2005) for an exposure time $\gtrsim 0.5$ Msec.

During two years of operation INTEGRAL provided huge amount of data. The deep observations of Galactic Center and Galactic Plane have been conducted with total exposure $>10 \mathrm{Msec}$, more then $2 \mathrm{Msec}$ have been dedicated to extragalactic fields. As seen from Fig. 1 the significant area of the sky is now covered by INTEGRAL observations.

\section{Work status and Future plans}

To perform all-sky survey we utilized data of our proprietary INTEGRAL/IBIS observations and publicly available data at the moment of August 2005. We used high Galactic latitude $\left(|b|>20^{\circ}\right)$ observations to prevent contamination from Galactic sources. The survey coverage versus limiting flux is shown on the left panel of Fig. 2. The survey area reaches its geometric limit of $24000 \mathrm{deg}^{2}$ for flux $>10^{-9} \mathrm{erg} \mathrm{s}^{-1} \mathrm{~cm}^{-2}$ and $50 \%$ of this area has sensitivity limit better then $6 \times 10^{-11} \mathrm{erg} \mathrm{s}^{-1} \mathrm{~cm}^{-2}$. The minimum detectable flux in the fields with highest exposure is $\backsim 10^{-11} \mathrm{erg} \mathrm{s}^{-1} \mathrm{~cm}^{-2}(\backsim \mathrm{mCrab})$.

Our hard X-ray source catalog contains more than 60 AGNs with ratio of both Seyfert types close to unity (will be published in subsequent paper). We used statistically clear sample (detections $>5.5 \sigma$ ) to derive number flux relation which is shown on the right panel of Fig. 2. The best-fit powerlaw slope is $1.30 \pm 0.07$. Estimates of the surface density at limiting flux of $\sim m C r a b$ give $(1.0 \pm 0.5) \times 10^{-2}$ sources per sq. degree. Regarding future prospects, therefore, of order of 400 hard X-ray sources should be detected from the whole sky. However, we should note that our sample may have bias due to source selection effect, because most of the used INTEGRAL extragalactic observations were pointed to the bright sources. To reduce this effect, the whole sky coverage is required. According to INTEGRAL observation program, systematic observations of extragalactic fields will be performed in year 2006 which will make it possible to obtain representative sample of AGNs. 
Nevertheless, the key information of the AGN evolution in local Universe rests on our knowledge of the volume number density and luminosity distribution of nearby AGN. The necessary distance information comes from identification of detected X-ray sources with host galaxies. The identification campaign is in progress (e.g. Sazonov et al., 2005) and full analysis of the whole source sample will be performed in the nearest future.

\section{Acknowledgements}

Work was partly supported by DFG Schwerpunkt programm "Witnesses of Cosmic History: Formation and evolution of black holes, galaxies and their environment".

\section{References}

Barthelmy, Scott D., et al. 2005, astro-ph/0507410, to be published in Space Science Reviews Boyle, B. J., Shanks, T., et al. 2000, MNRAS, 317, 1014

Fan, X., Strauss, M. A., Schneider, D. P., et al. 2001, AJ, 121, 54

Gehrels, N., Chincarini, G., Giommi, P., et al. 2004, ApJ 611, 1005

Giacconi, R. Zirm, A. Wang, J. Rosati, P., et al. 2002, ApJS, 139, 369

Giommi, P., Perri, M., \& Fiore, F. 1999, A\& A, 362, 799

Hasinger, G., Altieri, B., Arnaud, M., et al. 2001, AJ, 365, L45

Krivonos, R., Vikhlinin, A., Churazov, E., et al. 2005, ApJ, 625, 89

Lebrun, F., et al. 2003, A\&A, 411, 141

Levine, A. M., Lang, F. L., Lewin, W. H. G., et al. 1984, ApJS, 54, 581

Magorrian, J., et al. 2002, AJ, 105, 2285

Piccinotti, G., Mushotzky, R. F., Boldt, E. A., et al. 1982, ApJ, 253, 485

Revnivtsev, M., Sazonov, S., Jahoda, K., \& Gilfanov, M. 2004, A\&A, 418, 927

Sazonov, S., Churazov, E., Revnivtsev, M., et al. 2005, astro-ph/0508593, submitted to A\&A Letters

Steffen, A. T., Barger, A. J., Cowie, L. L., et al. 2003, ApJ, 611, L23

Tremaine, S., Gebhardt, K., et al. 2002, ApJ, 574, 740

Ubertini, P., Lebrun, F., Di Cocco, G., et al. 2003, A\&A, 411, L131

Ueda, Y., Akiyama, M., Ohta, K., \& Miyaji, T. 2003, ApJ, 598, 886

Ueda, Y., Takahashi, T., Ishisaki, Y., Ohashi, T., \& Makishima, K. 1999, ApJ, 524, L11

Winkler, C., et al. 2003, A\&A, 411, L1

Wood, K. S., Meekins, J. F., Yentis, D. J., et al. 1984, ApJS, 56, 507

\section{Discussion}

MACCARONE: How will the sensitivity of the INTEGRAL All-Sky Survey compare with the All-Sky Survey being conducted by SWIFT?

KrIvonos: The SWIFT Team announced very high sensitivity limit for high-latitude galactic fields. The sensitivity of less than $1 \mathrm{mCrab}$ will be reached in several days of observations of given area of the sky. However the actual sensitivity which can be reached by SWIFT will depend on how systematics will be overcome.

Due to smaller FOV, IBIS telescope has higher than BAT systematics limit. Thus, we have to wait for actual results from SWIFT survey to compare the sentivities. 